\section{Audiology \\ Neurotology}

Audiol Neurotol 2020;25:35-41

DOI: $10.1159 / 000504595$
Received: August 12, 2019

Accepted after revision: November 5, 2019

Published online: January 10, 2020

\title{
The Superiority of the Otolith System
}

\author{
Angel Ramos de Miguel ${ }^{a, b} \quad$ Andrzej Zarowski ${ }^{c}$ Morgana Sluydts ${ }^{c}$ \\ Angel Ramos Macias ${ }^{a, b}$ Floris L. Wuyts ${ }^{d}$
}

a Department of Otolaryngology, and Head and Neck Surgery, Complejo Hospitalario Universitario Insular Materno Infantil de Gran Canaria, Las Palmas de Gran Canaria, Spain; ${ }^{b}$ Department of Otolaryngology, Faculty of Medicine, University of Las Palmas de Gran Canaria (ULPGC), Hearing and Balance Laboratory, University of Las Palmas de Gran Canaria (ULPGC), Las Palmas de Gran Canaria, Spain; ' European Institute for Otorhinolaryngology, GZA Hospitals Antwerp, Wilrijk, Belgium; ${ }^{\mathrm{d}}$ Lab for Equilibrium Investigations and Aerospace, University of Antwerp, Antwerp, Belgium

\section{Keywords}

Otolith function - Vestibular implant · Balance $\cdot$ Utricle .

Saccule $\cdot$ Vestibulo-ocular reflex $\cdot$ Ocular counter roll

\section{Abstract}

Background: The peripheral vestibular end organ is considered to consist of semi-circular canals (SCC) for detection of angular accelerations and the otoliths for detection of linear accelerations. However, otoliths being phylogenetically the oldest part of the vestibular sensory organs are involved in detection of all motions. Summary: This study elaborates on this property of the otolith organ, as this concept can be of importance for the currently designed vestibular implant devices. Key Message: The analysis of the evolution of the inner ear and examination of clinical examples shows the robustness of the otolith system and inhibition capacity of the SCC. The otolith system must be considered superior to the SCC system as illustrated by evolution, clinical evidence, and physical principles.

(c) 2020 The Author(s)

Published by S. Karger AG, Basel

\section{KARGER}

E-Mail karger@karger.com www.karger.com/aud

Karger

Open access

\section{(C) 2020 The Author(s)}

Published by S. Karger AG, Basel

This article is licensed under the Creative Commons AttributionNonCommercial-NoDerivatives 4.0 International License (CC BYNC-ND) (http://www.karger.com/Services/OpenAccessLicense) Usage and distribution for commercial purposes as well as any distribution of modified material requires written permission.

\section{Introduction}

The peripheral vestibular system consists of the semicircular canals (SCC) and the otoliths. In general, it is conceived that SCC and otoliths are complementary, since the SCC detect angular accelerations [Fernandez and Goldberg, 1971], whereas the otoliths detect linear accelerations [Fernandez and Goldberg, 1976] including gravity. In this study, we will build up a rationale for extending this standard view. In essence, the otoliths detect all accelerations, i.e., angular and linear accelerations, and thus can be considered as the core motion detectors.

\section{Evolution}

Throughout evolution of life on Earth, gravity and more specifically $1 \mathrm{~g}$, gravitational acceleration on Earth of about $9.8 \mathrm{~m} / \mathrm{s}^{2}$, has been the sole constant factor, in contrast to environmental conditions such as temperature, humidity, pressure, oxygen level, etc.

Detection of gravity appears to be essential for complex life. Even plants have evolved multiple mechanisms
Angel Ramos de Miguel, PhD, Department of Otolaryngology

Faculty of Medicine, University of Las Palmas de Gran Canaria (ULPGC)

Avda. Maritima del Sur s/n

ES-35016 Las Palmas de Gran Canaria (Spain)

E-Mail aramos.gcc@gmail.com 


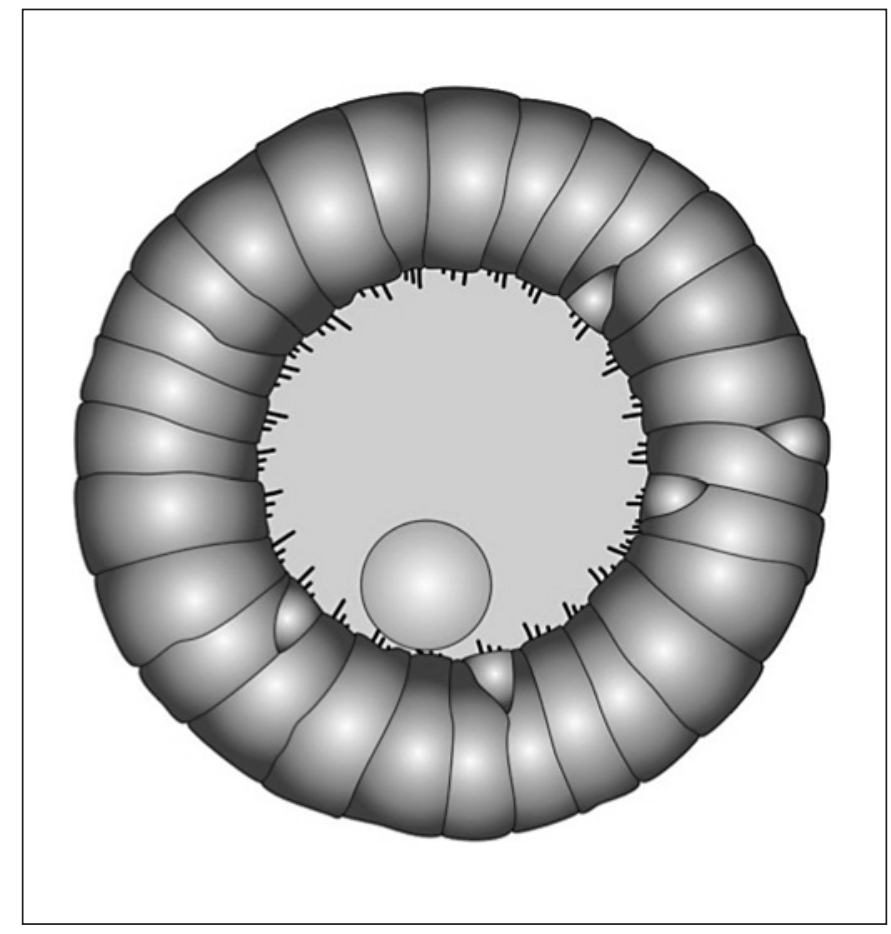

Fig. 1. Statocyst consists of a sac-like structure containing a statolith, a mineralised mass.

to sense gravity. Gravitropism directs plants growth relative to gravity. It determines the orientation and the final architecture of the plant.

Flowering plants, for example, sense gravity via specialized cells termed statocytes [Fukaki et al., 1998; Kiss, 2000]. Within these statocytes, dense starch-filled organelles (statoliths) settle relative to the gravity vector, providing directional information to the plant [MacCleery et al., 1999; Saito, 2005]. In case of a reorientation of the plant, the settling of the statoliths starts a biochemical cascade to promote differential growth in the elongation zone of the plant root or shoot.

Moving to the animal kingdom, it seems that the simplest animals, like jellyfish, also have statoliths to perceive gravity as well as water current. Jellyfish appeared in the Ediacaran Period 635 million years ago. Approximately 100 million years later in the Cambrian Period, deuterostome (like sea cucumber) appeared and they have a statolith located in the statocyst as balance system (Fig. 1).

With the arrival of the chordate animals, of which vertebrates is a subgroup, jawless fish with a single SCC and a macula appeared around 419 million years ago. This is the seed for the development of the inner ear as it is known today (Fig. 2). Still there are living animals, like the hagfish, with only one SCC and a common macule [Higuchi et al., 2019].

Lungfish, or dipnoans, appeared 400 million years ago, with 3 SCC and 3 otoliths (utricle, saccule, and lagena). However, there was no cochlea.

From vertebrates, tetrapod appears and then amniote 320 million years ago. At this point, the synapsida (future mammals) and sauropsida (reptiles like dinosaur and birds) appeared. Figure 3 represents the inner ear of a Tyrannosaurus rex, dating 68 million years ago [Witmer et al., 2009]. Mammals appeared around 200 million years ago with the currently known cochleo-vestibular system.

The third otolith system, i.e., the lagena, is still present in many animals (all descendants of the sauropsida). Crocodiles do not have a real cochlea but their saccule and lagena serve for both hearing and balance, and their utricle only serves for balance [Walsh et al., 2009].

The lagena of some birds and fish has a high concentration of metals that allows to use the lagena as a magnetometer (compass) to feel the Earth's geomagnetic field [Harada et al., 2001].

Summarizing the evolution of the inner ear, the otolith organ emerged in jellyfish 635 million years ago, the SCC 400 million years ago, whereas the coiled cochlea appeared "only" 200 million years ago (Fig. 4). So actually, the vestibular system is not part of the inner ear, but it is the other way around.

\section{The Vestibular System}

The aim of the vestibular system as it has evolved up to date is to provide information of balance, self-motion, and position in space. Hereto, the vestibular information about motion and position is integrated with vision and proprioception.

\section{Semi-Circular Canals}

The geometrical configuration of the SCC is basically a torus with an embedded deflector (cupula). The sensorial epithelium in the SCC are the hair cells at the base of the cupula, which is a membrane that deflects driven by the inertial mass of the endolymph upon angular acceleration, i.e., rotation. The dynamics of the system can be deduced from hydrodynamic principles [Van Egmond et al., 1949; Jones and Spells, 1963; Mayne, 1965; Steer Jr, 1967]. Essentially, when the head starts to rotate, the inertia of the endolymph causes a
Ramos de Miguel/Zarowski/Sluydts/ Ramos Macias/Wuyts 


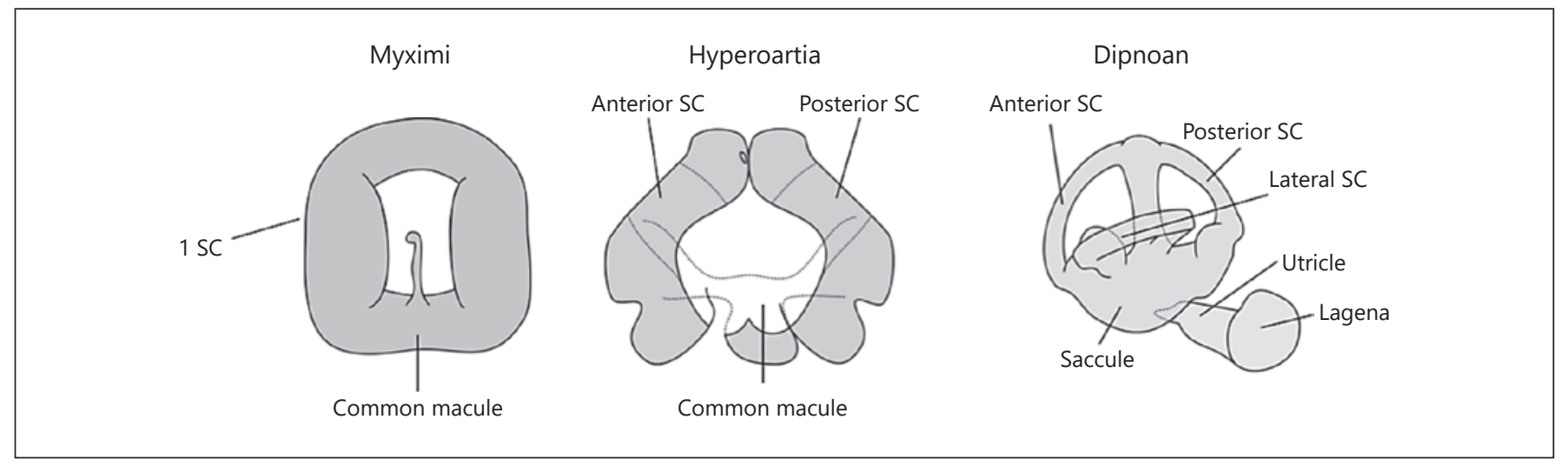

Fig. 2. Evolution of SCC. 1 SCC appears in Myximi, then 2 SCC with Hyperoartia, and finally 3 SCC with Dipnoan. Image adapted from Higuchi et al. [2019].

Fig. 3. Inner ear of a Tyrannosaurus rex. Similarities can be observed with crocodile and pigeon that also evolve from Sauropsida [Witmer et al., 2009; Walsh et al., 2009].

The Superiority of the Otolith System

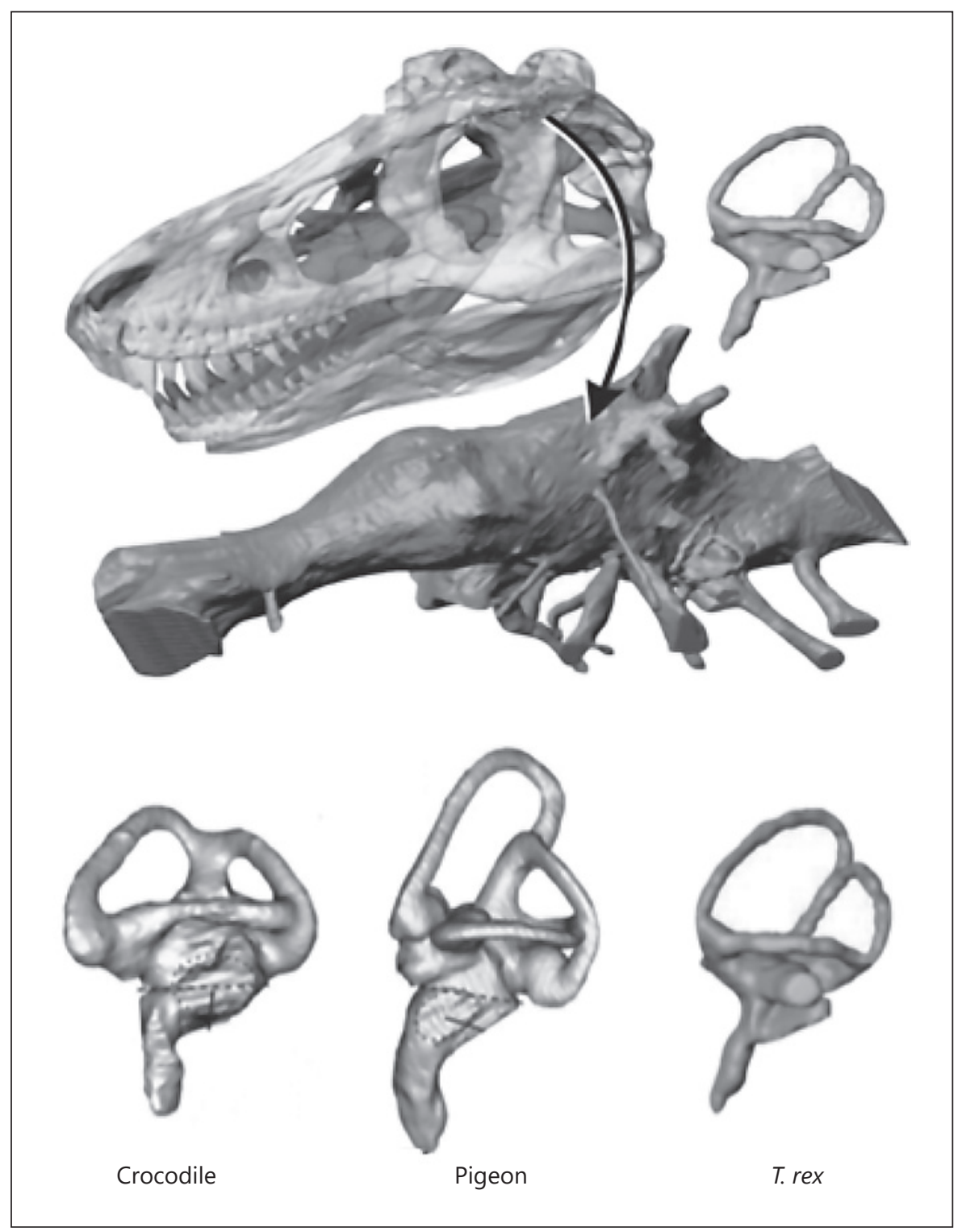

Audiol Neurotol 2020;25:35-41 DOI: $10.1159 / 000504595$ 


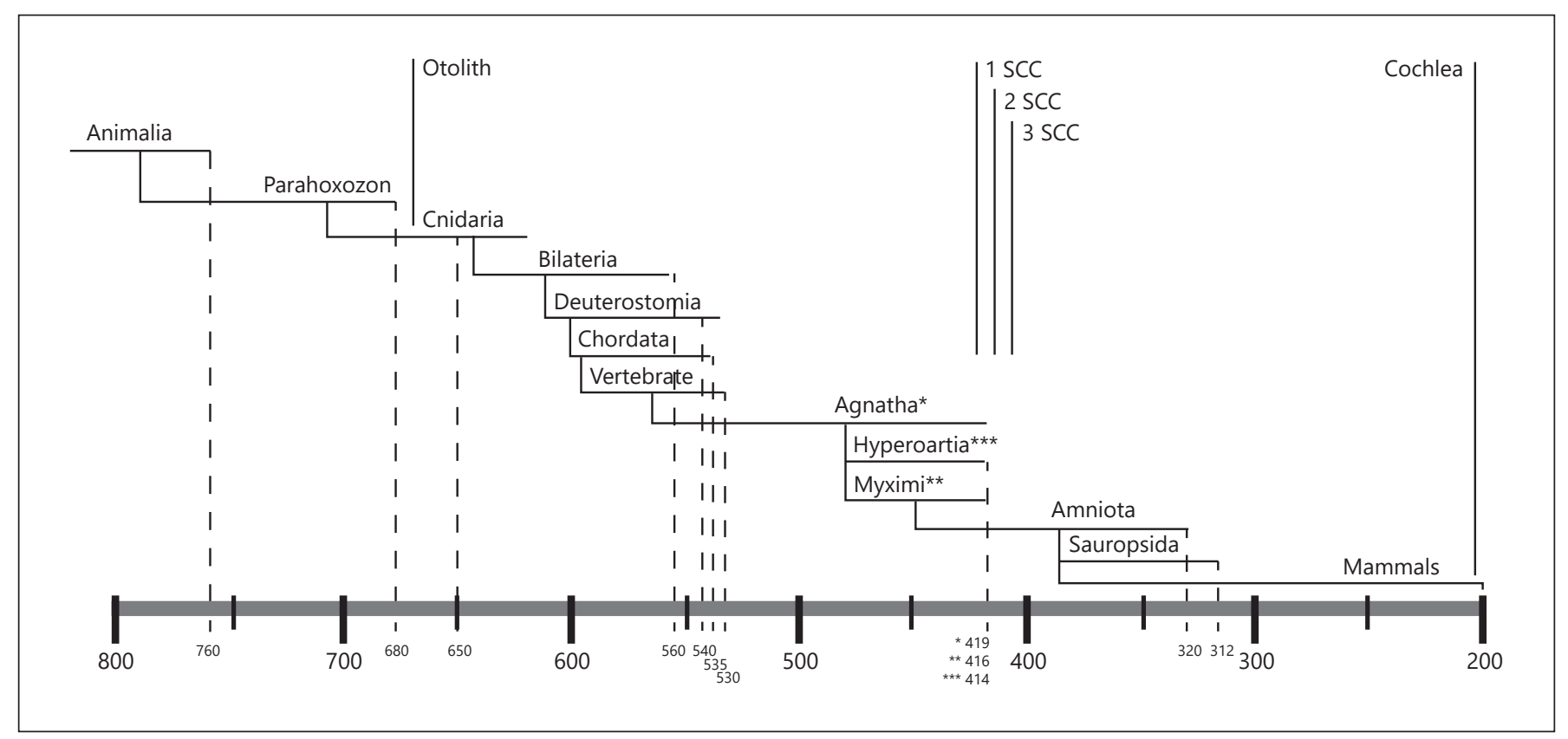

Fig. 4. Summary of the chronological timeline of the inner ear evolution.

deflection of the cupula. This deflection depends on the direction and amplitude of the angular acceleration, and eventually it is proportional to the velocity of the head movement. The derived formulas on the movement of the cupula and the concomitant deflection of the hair cell cilia start from the principle that the centre of rotation is located in the centre of the SCC. However, the canals are located eccentrically. In case of Caucasian adults, the distance between both lateral SCC is $8.87 \pm$ $0.06 \mathrm{~cm}$ [Nowé et al., 2003]. Hence, upon rotation of the head around the craniocaudal axis, the SCC perceive additional tangential acceleration of the endolymph and the cupula. This tangential acceleration equals to the angular acceleration multiplied by the distance to the centre of rotation. But this is merely a scaling factor. Sustained rotation will be sensed only for approximately $20 \mathrm{~s}$ after cessation of the angular acceleration by means of the velocity storage mechanism (VSM). The VSM is activated by both visual and vestibular rotation cues and is modified by gravity. The network of neurons in the superior vestibular nucleus and medial vestibular nucleus as well as their commissural interconnections are critical for producing velocity storage [Yakushin et al., 2017]. One of the proposed function is to prolong the vestibulo-ocular reflex (VOR) longer than the mechanically governed $10 \mathrm{~s}$, which is the time constant of this damped oscillator. During the rotation (i.e., the acceleration phase and the sustained constant rotation), the otoliths are sensing this movement. This is a consequence of the highly specialized design of the otolith system that is driven by million years of evolution to detect gravity.

\section{Otoliths}

The saccule and utricle are complex curved maculae that can detect movement in virtually all directions. Highly simplified, one can represent the otolith organ as 3 layers on top of each other (Fig. 5). The base layer contains the sensorial epithelium with the hair cells. The cilia of the hair cells protrude in the second layer, being a membrane on top of which lie the otoconial calcium carbonate crystals forming the third layer. These so called statoliths, have a density of $2.71 \mathrm{~g} / \mathrm{cm}^{3}$ [Carlström, 1953] that is much heavier than the surrounding endolymph (density $\sim 1 \mathrm{~g} / \mathrm{cm}^{3}$ ) and membrane. Any linear acceleration will exert a shear force on the otoconial layer and cause a displacement relative to the base layer and consequently causing a change in the membrane potential of the hair cells, which then results in a complex pattern of simultaneous excitation and inhibition.

Although the triggering stimulus for the otoliths is a shear force on the otoconial layer, this force can emerge both by angular as well as linear accelerations. The following rationale will clarify this concept.
Ramos de Miguel/Zarowski/Sluydts/ Ramos Macias/Wuyts 


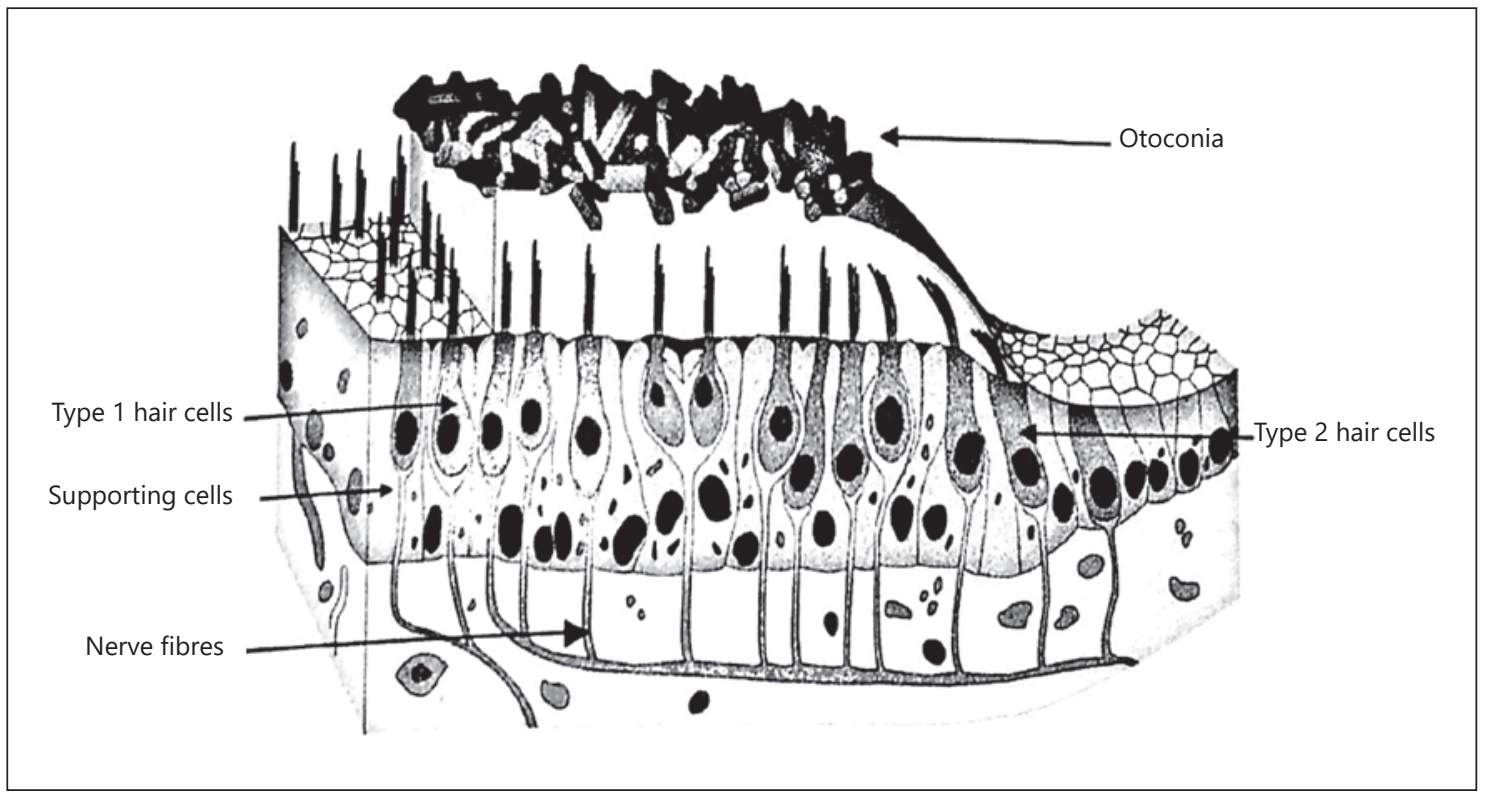

Fig. 5. Macula of the utricle with the calcite crystal or otoconia.



Fig. 6. Complete HIT reflexes during left rotation. Left: healthy labyrinths produce only VOR due to the cancelation of OCR on both sides. Right: unilateral otolith disfunction produce VOR and also OCR to the damaged side.

Indeed, as proven by the evolutionary pathway, both in plants as well as in animals, gravity detection is the core function of the otolith system. Gravity detection ensures optimal growth, upright posture, balanced locomotion, and detects deviations from the vertical, including falls, for example.

The Superiority of the Otolith System

\section{Clinical Example of Multidimensionality}

During a short head rotation to the left, both SCC left and right are stimulated giving rise to the VOR (Fig. 6, left). The output of the left and right SCC is antagonistic, however, with the left SCC giving rise to an increase in

Audiol Neurotol 2020;25:35-41 
spike rate of the hair cells, and the right SCC generating a decrease in spike rate. The left and right vestibular nuclei in the brainstem decode these antagonistic signals from the left and right SCC to drive the respective left oculomotor nucleus and right abducens nucleus that generate compensatory eye movements with eyes going to the right mediated by the respective medial and lateral eye muscles. At the same time, both utricles are stimulated with identical shear forces pulling the otoconial layers outward, because of the centrifugal force that is pointing out from the centre of rotation. The left utricle will then generate a clockwise (from the point of view of the subject) ocular counter roll (OCR) and the right utricle will give rise to a counter clockwise OCR. However, both cancel each other out, generating no net eye movement. But, in case of one deficient labyrinth, this would cause a slight net OCR (Fig. 6, right). For a typical head rotation of 200 degrees per second, as produced during the Head Impulse Test (HIT), this would generate an OCR of 0.4 degrees, given an OCR gain of $15 \%$, which is negligible and hardly detectible with the current methods. Based on this, HIT is the combination of VOR and OCR. In the case of healthy peripheral vestibular system, response during a HIT test is purely VOR. But in the case of one deficient otolith, a small OCR will appear.

This does not imply that the utricles do not contribute to the whole of the reflex. They even send their signal to the respective cortical areas like the operculum parietale 2 (OP2) in the brain, where the SCC signals converge with the otolith signals to appropriately interpret the whole movement stimulus [zu Eulenburg et al., 2012]. For example, in case of tilt-translation ambiguity, otoliths respond identically to translational (inertial) accelerations and changes in head orientation relative to gravity. This sensory ambiguity can be resolved using SCC signals. This procedure is done in the vestibular nuclei, rostral fastigial nuclei, cerebellar nodulus/uvula, and thalamus [Angelaki and Yakusheva, 2009].

Additionally, the otolith system is multidimensional, and there is no left right agonist/antagonist mechanism as in the canal system. This is a very important property because even with one otolith system, normal OCR reflexes are generated upon lateroflexion, showing the robustness of the otolith system [Wuyts et al., 2001]. The otoliths also preserve autonomic function as recently demonstrated in cosmonauts [Hallgren et al., 2016]. The otolith system mediates the VSM through the so-called otolith dump, which is the abolishment of the postrotatory nystagmus by inclination of the head. This is commonly adopted by figure skaters after high-speed pirou- ettes [Hain et al., 1988]. Otolith input in general inhibits the SCC signals [Hain et al., 1988]. The otoconial membrane neither has a damped spring configuration like the SCC, and hence the otoliths permanently sense the gravity vector. Therefore, based on evolution, robustness and inhibition capacity, the otolith is superior to the canal system.

Clinical observations show that, except for Tumarkin syndrome, almost no other vestibular disorders can be attributed solely to unilateral otolith disfunction. Even during a vestibular neuritis, which affects the superior vestibular nerve (and thus the signals coming from the horizontal and anterior SCC and the utricle), the complaint of the patient is spinning vertigo with a dominant horizontal nystagmus with a slight torsional component, however, without sensations of the Tumarkin type. Neither can other complaints of dizziness or vertigo be attributed to an otolith disorder, despite the absence of vestibular evoked myogenic potentials in some cases. Thus, it can be concluded that isolated otolith deficits are difficult to identify, perhaps because there are few to none. Even if one otolith system fails, the other otoliths are sufficient to ensure gravity detection. So only in case of bilateral total vestibulopathy, there will be a lack of gravity detection. Hence, the necessity to restore, in the first place, the otolith function and this by means of a vestibular implant, for example. Moreover, based on the abovementioned evidence, unilateral restoration should be sufficient.

\section{Conclusion}

The otolith system must be considered superior to the SCC system as illustrated by evolution, clinical evidence, and physical principles. Hence, when replacing the vestibular system by means of a vestibular implant in a patient with bilateral vestibular areflexia, we hypothesize to target at first the otolith system.

\section{Statement of Ethics}

The authors have no ethical conflicts to disclose.

\section{Disclosure Statement}

All authors declare that they have no conflicts of interest.
40

Audiol Neurotol 2020;25:35-41 DOI: $10.1159 / 000504595$
Ramos de Miguel/Zarowski/Sluydts/ Ramos Macias/Wuyts 


\section{Funding Sources}

This project has received funding from the European Union's Horizon 2020 Research and Innovation Programme under Grant Agreement No. 801127 (BionicVest).

\section{Author Contributions}

Angel Ramos de Miguel: hypothesis proposal, design of the work, drafting of the manuscript. Andrzej Zarowski: drafting and reviewing of the manuscript. Morgana Sluydts: clinical examples, as well as drafting and reviewing of the manuscript. Angel Ramos Macias: drafting and reviewing of the manuscript. Floris L. Wuyts: clinical support of the hypothesis, design of the work, drafting of the manuscript.

\section{References}

Angelaki DE, Yakusheva TA. How vestibular neurons solve the tilt/translation ambiguity. Comparison of brainstem, cerebellum, and thalamus. Ann N Y Acad Sci. 2009 May; 1164(1):19-28

Carlström D, Engström H, Hjorth S. Electron microscopic and $\mathrm{x}$-ray diffraction studies of statoconia. Laryngoscope. 1953 Nov;63(11) $1052-7$.

Fernández C, Goldberg JM. Physiology of peripheral neurons innervating otolith organs of the squirrel monkey. I. Response to static tilts and to long-duration centrifugal force. J Neurophysiol. 1976 Sep;39(5):970-84.

Fernandez C, Goldberg JM. Physiology of peripheral neurons innervating semicircular canals of the squirrel monkey. II. Response to sinusoidal stimulation and dynamics of peripheral vestibular system. J Neurophysiol. 1971 Jul;34(4):661-75.

Fukaki H, Wysocka-Diller J, Kato T, Fujisawa H, Benfey PN, Tasaka M. Genetic evidence that the endodermis is essential for shoot gravitropism in Arabidopsis thaliana. Plant J. 1998 May;14(4):425-30.

Hain TC, Zee DS, Maria BL. Tilt suppression of vestibulo-ocular reflex in patients with cerebellar lesions. Acta Otolaryngol. 1988 JanFeb;105(1-2):13-20.

Hallgren E, Kornilova L, Fransen E, Glukhikh D, Moore ST, Clément G, et al. Decreased otolith-mediated vestibular response in 25 astronauts induced by long-duration spaceflight. J Neurophysiol. 2016 Jun;115(6):3045-51.
Harada Y, Taniguchi M, Namatame H, Iida A. Magnetic materials in otoliths of bird and fish lagena and their function. Acta Otolaryngol. 2001 Jul;121(5):590-5.

Higuchi S, Sugahara F, Pascual-Anaya J, Takagi W, Oisi Y, Kuratani S. Inner ear development in cyclostomes and evolution of the vertebrate semicircular canals. Nature. 2019 Jan; 565(7739):347-50.

Jones GM, Spells KE. A theoretical and comparative study of the functional dependence of the semicircular canal upon its physical dimensions. Proc R Soc Lond B Biol Sci. 1963 Mar; 157(968):403-19.

Kiss JZ. Mechanisms of the early phases of plant gravitropism. CRC Crit Rev Plant Sci. 2000; 19(6):551-73.

MacCleery SA, Kiss JZ. Plastid sedimentation kinetics in roots of wild-type and starch-deficient mutants of Arabidopsis. Plant Physiol. 1999 May;120(1):183-92.

Mayne R. The "match" of the semicircular canals to the dynamic requirements of various species. In: Symposium on the Role of the Vestibular Organs in the Exploration of Space. Washington, D.C.: National Aeronautics and Space Administration, SP-77, 1965, p. 57-67

Nowé V, Wuyts FL, Hoppenbrouwers M, Van de Heyning PH, De Schepper AM, Parizel PM. The interutricular distance determined from external landmarks. J Vestib Res. 2003;13(1): $17-23$.
Saito C, Morita MT, Kato T, Tasaka M. Amyloplasts and vacuolar membrane dynamics in the living graviperceptive cell of the Arabidopsis inflorescence stem. Plant Cell. 2005 Feb;17(2):548-58.

Steer RW Jr. The influence of angular and linear acceleration and thermal stimulation on the human semicircular canal [doctoral dissertation]. Cambridge (MA): Massachusetts Institute of Technology; 1967.

Van Egmond AA, Groen JJ, Jongkees LB. The mechanics of the semicircular canal. J Physiol. 1949 Dec;110(1-2):1-17.

Walsh SA, Barrett PM, Milner AC, Manley G, Witmer LM. Inner ear anatomy is a proxy for deducing auditory capability and behaviour in reptiles and birds. Proc Biol Sci. 2009 Apr; 276(1660):1355-60.

Witmer LM, Ridgely RC. New insights into the brain, braincase, and ear region of tyrannosaurs (Dinosauria, Theropoda), with implications for sensory organization and behavior. Anat Rec (Hoboken). 2009 Sep;292(9):1266-96.

Wuyts FL, Van der Stappen A, Hoppenbrouwers M, Van Dyck D, Schor RH, Furman JM, et al. Otolith function after acoustic neuroma surgery. Acta Otolaryngol Suppl. 2001;545:170-3.

Yakushin SB, Raphan T, Cohen B. Coding of Velocity Storage in the Vestibular Nuclei. Front Neurol. 2017 Aug;8:386.

zu Eulenburg P, Caspers S, Roski C, Eickhoff SB. Meta-analytical definition and functional connectivity of the human vestibular cortex. Neuroimage. 2012 Mar;60(1):162-9. 